\title{
Modern cataract surgery in a highly myopic population
}

\author{
K A GROSS and J L PEARCE
}

From Bromsgrove General Hospital, Worcester B60 2BG

SUMMARY A retrospective series of 117 highly myopic eyes with extracapsular cataract extractions and posterior chamber lens implants is presented. The results are analysed with reference to the current understanding of the complications anticipated. Of particular note is the use of lowpowered posteriorly vaulted posterior chamber implants, with and without primary posterior capsulotomy. The results show a low retinal detachment rate (one eye, mean follow-up 12 months); low induced astigmatism (average $0.86 \mathrm{D}$ ); low myopic postoperative refraction (median $-1.0 \mathrm{D})$; and high patient satisfaction.

Irvine, ${ }^{1}$ in his article on the pathogenesis of aphakic retinal detachments, reminds us of A C Hilding's work of 1954 on the inertial vitreous traction inherent in posterior vitreous detachment and high velocity ocular saccades. His article argues the importance of the posterior convexity of the lens in stabilising the vitreous. However, in his assessment of modern cataract surgery he does not discuss the results of posteriorly vaulted posterior chamber lenses following extracapsular cataract extraction.

To explore the possible advantages of these lens implants a retrospective review was made of all cataract surgery performed in the Mid Worcester District Health Authority over the years 1982-4 inclusive.

\section{Materials and methods}

\section{POPULATION}

During this three-year period 1235 cataract extractions were performed in the district. In 117 of these a calculated intraocular lens implant (IOL) of $\leqslant 16 \cdot 0$ dioptres (D) was fitted. In a further 89 cases lenses with powers of $16-18.0 \mathrm{D}$ were used. It should be noted that the posterior vaulting and biconvex form of the optic mean that the lens's nodal point is placed $5 \cdot 0-5 \cdot 1 \mathrm{~mm}$ from the corneal surface. A comparable 'iris plane' lens would have a 3.5-4.0 D lower power. We are thus discussing a sample of highly myopic eyes.

\section{SAM PLE}

The notes of all 117 patients were reviewed at a Correspondence to K A Gross, FRCS, 14 Crondal Place, Edgbaston, Birmingham B15 2LB. maximum of 44 months and a minimum of 9 months after operation. Their average age was 69.2 years, with a maximum of 90 and a median of 71 years. There were two notably younger patients (17 and 23 years), one having Down's syndrome, the other having congenital cataract with nystagmus.

The preoperative corrected visual acuity with high myopic refraction was $6 / 36$ or less in $48 \%$ of patients. A subgroup of 16 patients had vision of 6/12 or better. All had rapidly advancing myopic nuclear sclerosis, focal posterior cataracts, or contralateral pseudophakos. The mean spherical equivalent correction averaged $-10.0 \mathrm{D}$, with a standard deviation of 1.08 D (Fig. 1).

The average axial length in this group of patients was $27.34 \mathrm{~mm}$, SD $0.15 \mathrm{~mm}$. The maximum measurement was $32.3 \mathrm{~mm}$. In one patient the axial length was $23.6 \mathrm{~mm}$, and the eye was implanted with a 16.0 $\mathrm{D}$ lens owing to an abnormally high corneal refractive power. This patient was eliminated from the follow-up as he would not carry the same high risk factors associated with axial myopic cataract extraction. The minimum axial length was thus $24.9 \mathrm{~mm}$ (Fig. 2).

\section{SURGERY}

All patients underwent non-automated extracapsular cataract extraction with no peripheral iridectomies. Sodium hyaluronate $1 \%$ (Healonid) was used in all operations. $1 \mathrm{ml}$ of $0.75 \%$ bupivacaine retrobulbar injection (in addition to general anaesthesia) was given routinely, though six patients were limited to a local anaesthetic alone in view of their poor general health. $73 \%$ received primary posterior capsulotomies prior to the insertion of the posteriorly vaulted, 


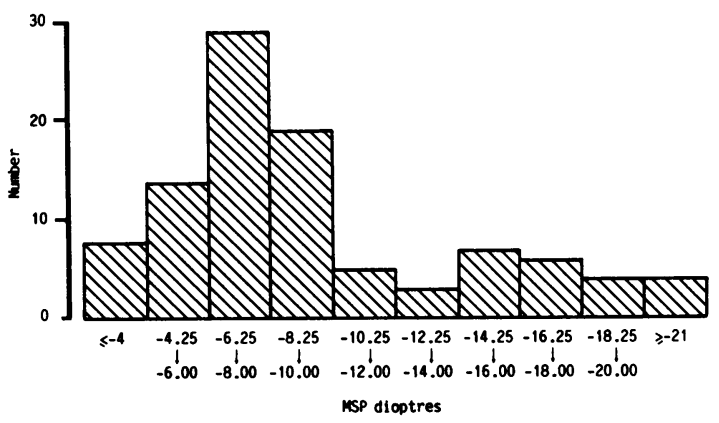

Fig. 1 Distribution of the mean spherical equivalents of the preoperative refraction.

posterior chamber lenses, which included $63 \mathrm{~J}$ Pearce tripods, $43 \mathrm{~J}$ Pearce Y loops, and nine other miscellaneous posterior vaulted IOL. Twenty-eight patients underwent bilateral cataract surgery, and five patients underwent a combined cataract/ trabeculectomy procedure.

The powers of the lenses used are shown in Fig. 3. These were influenced by availability, particularly the absence of lenses of less than 4 dioptres at that time.

Patients were routinely discharged the following day on guttae dexamethasone 2-hourly, with miotics if necessary. It should be noted that no eye received prophylactic retinal treatment (cryotherapy etc.) prior to surgery.

\section{Results}

A minimum period of three months documented follow-up was specified. This eliminated seven patients owing to: death (1), return abroad (2 patients with favourable reports by letter subsequently), failure to attend (2), and return to distant UK homes (2). Two further patients were eliminated owing to abandoned implantation peroperatively.

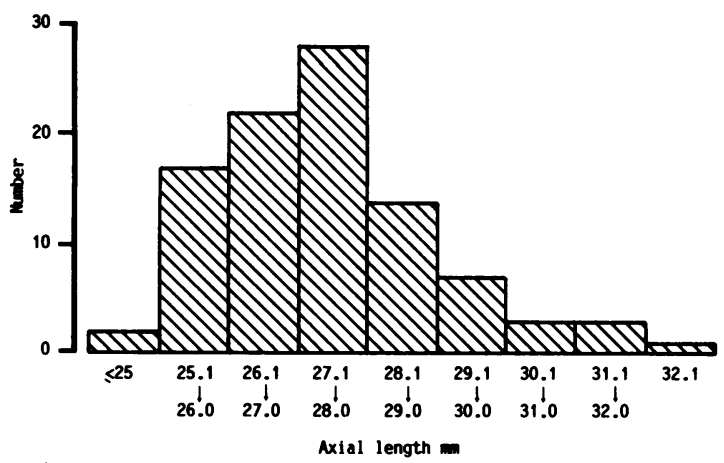

Fig. 2 Axial lengths as recorded with various $A$ scan ultrasounds.

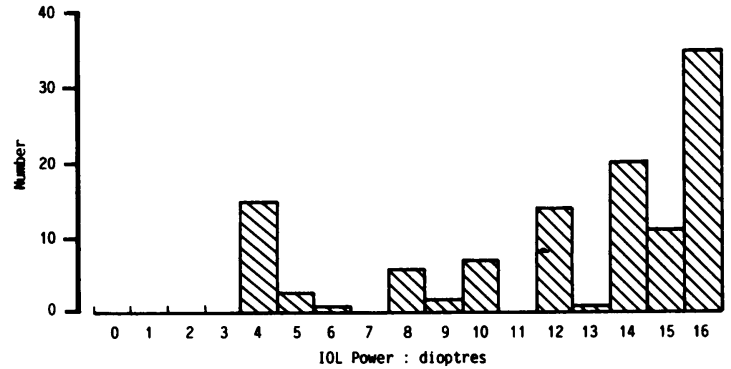

Fig. 3 Distribution of intraocular lens's powers used in these patients. Note peak at 4.0 D due to the lack of available low power lenses at that time.

One patient suffered a posterior dislocation of the implant into the vitreous at four days (vision $6 / 6$ after 12 months with no additional complications). With the patient eliminated previously (axial length of 23.6 $\mathrm{mm}$ ), this left 106 patients for analysis.

The average period of follow-up for which full notes were available was 12.3 months, with a maximum of 36 , a minimum of 3 , a median of 12 , and a standard deviation of $1 \cdot 14$. Thus there were 54 patients with a greater than 12-month follow-up (average 19.5 months).

The visual acuity of these patients, who were lifelong myopes, improved to $\geqslant 6 / 12$ without glasses in $40.5 \%$ of cases; $83.6 \%$ achieved a vision in excess of 6/12 with glasses (Figs. 4, 5). Direct comparison with the levels of vision achieved in uncomplicated cataract surgery $\left(93+\% \geqslant 6 / 12^{23}\right)$ is not possible in view of the degenerate retinal function in high myopia.

Eleven patients achieved $\leqslant 6 / 36(8.6 \%)$, and it is conspicuous that nine of this group $(81.8 \%)$ had an axial length $>28 \mathrm{~mm}$ (in the whole sample only $28 \%$ had an axial length $>28 \mathrm{~mm}$ ). This was a significant finding (Student's $t$ test, $\mathrm{p} \ll 0.01$ ). Nine of these patients had gross preoperative myopic macular degeneration (or senile macular degeneration), two with coexistent chronic simple glaucoma (CSG), and

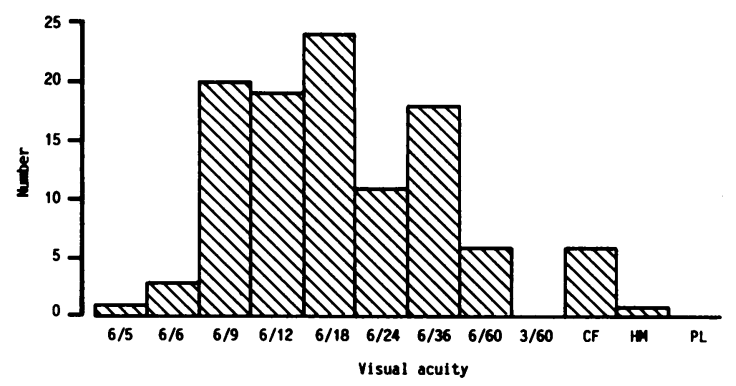

Fig. 4 Visual acuity recorded without glasses at one month after operation. 


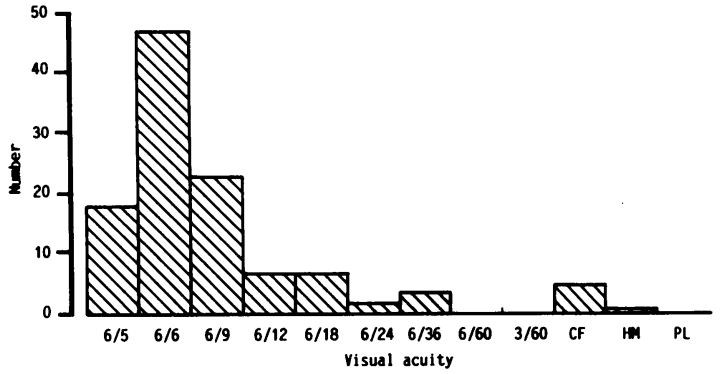

Fig. 5 Visual acuity recorded with glasses at more than three months after surgery.

one had CSG alone. One patient had proliferative diabetic retinopathy despite the relative protection that myopia is believed to afford to diabetic fundi. ${ }^{45}$

The mean spherical equivalent of the postoperative correction at more than three months, averaged $-0.76 \mathrm{D}$ with a median of $-1.0 \mathrm{D} .69 .5 \%$ had $\leqslant 2.0 \mathrm{D}$ spread around the desired postoperative refraction of $-1.0 \mathrm{D}$ (Fig. 6). $10 \cdot 5 \%$ had a $>3.0 \mathrm{D}$ error range. It is perhaps noteworthy that not all patients could be calculated for the optimum optical result ${ }^{6}$ owing to the existing refraction of the other eye and/or the availability of specific lens powers. A minor trend of increasing myopia during the first month after operation was found to be statistically not significant.

Surgical astigmatism averaged $0.86 \mathrm{D}$ at three months plus, after operation. $72.5 \%$ had a cylinder of $<1.0 \mathrm{D}$, and $6 \%$ had $>2.0 \mathrm{D}$. A significant $(\mathrm{p} \ll 0.05)$ decrease in astigmatism over the first three months was noted. $91 \%$ of eyes were noted to have the posterior capsule sealed against the posteriorly convex implant surface, with or without a primary capsulotomy.

\section{COMPLICATIONS}

The peroperative problems were restricted to the two cases mentioned in which implantation was abandoned because of posterior capsular tears and vitreous loss. Of these one has $<6 / 60$ due to myopic

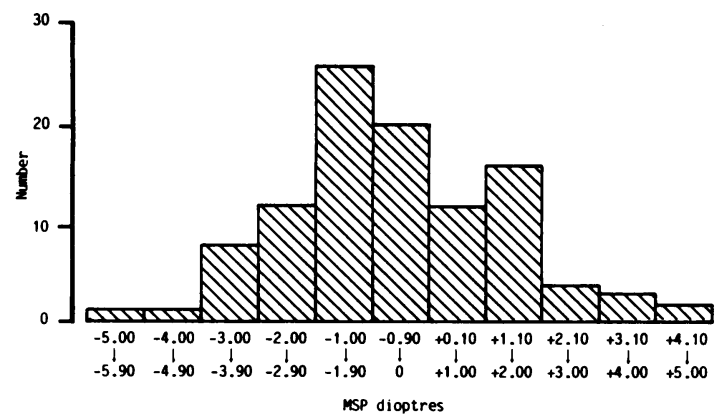

Fig. 6 Distribution of mean spherical equivalents of the postoperative refraction. degeneration, but has been followed up for 18 months without further complications. The other has had a two-year follow-up with $6 / 9$ corrected vision. In the immediate postoperative period there were minor complications of residual soft lens matter (6), small hyphaemata (2), and small anterior chamber vitreous prolapses (4). These complications showed a decreasing incidence with greater surgical experience, and 10 out of the 12 achieved $>6 / 12$ vision.

One patient suffered a posterior dislocation of the implant into the inferior vitreous within four days of surgery. There have been no additional complications in this patient, who retained $6 / 6$ vision at 12 months follow-up, using a contact lens.

Long-term complications included opacification of the posterior capsule in 11 patients, sufficient to require a secondary capsulotomy. Six of these had had what was presumably a non-axial, primary capsulotomy. There were no cases of pupil trap, presumably owing to the posterior vaulting of the IOL giving a good clearance from the miosed pupil. Some distortion of the pupil was noted in three cases from anterior capsule tags or vitreous. One patient developed decentration of the posterior chamber lens, secondary to capsular fibrosis, although the optic remained in the visual axis, permitting near normal function.

There have been no cases of 'clinical cystoid macular oedema' (CMO), infection, severe inflammation, or corneal decompensation.

There has been one case of postoperative retinal detachment. This occurred in a patient who had had no obvious surgical problems. He presented with a posterior, large U-shaped retinal tear in lattice degeneration following a posterior vitreous detachment occurring 18 months postoperatively. Simple radial plombage has reattached the retina with retained vision of $6 / 12$. This is the pattern of a phakic as opposed to an aphakic retinal detachment (both in appearance and in response to treatment). The good surgical prognosis of retinal detachment surgery following extracapsular cataract extraction with posterior chamber lenses has been previously noted. $^{78}$

\section{Discussion}

This sample, extracted from the cataract surgery performed over a three-year period, was designed to be among the highest-risk surgical population. A multitude of surgical series demonstrate the greatly increased risk of aphakic retinal detachment in axial myopia. $^{-12}$

The poor Snellen visual acuities achieved in nine of 13 patients with preoperatively identified 'myopic/ senile' degeneration should not obscure the very real 
improvement in these patients' undistorted peripheral vision. In addition four of the 13, who might have been denied surgery because of their known macular degeneration, achieved better than $6 / 18$.

Irvine $^{1}$ has eloquently discussed the various known mechanisms of aphakic retinal detachment, notably the inertial vitreous traction present with horizontal ocular saccades. He summarises the results of different surgical techniques, but stops short of discussing posterior vaulted, posterior chamber implants. This is the very modification most likely to reproduce the vitreous support of the posterior convexity of the crystalline lens.

His article also comments on the increased risk of retinal detachment with primary posterior capsulotomy, possibly due to loss of hyaluronic acid from the vitreous ${ }^{13}$ and unplugging of small retinal holes. The intraocular lenses used in this series of patients, being posteriorly vaulted and having a posteriorly convex optic, achieved a 'seal' against the posterior capsule (and capsulotomy) in $91 \%$ of cases. These lenses have now been in use for more than five years, and our experience of them warrants wider discussion.

The low incidence of aphakic retinal detachment (ARD) in this group of patients so far is very reassuring, as $67.7 \%$ of such detachments occur in the first year after operation (cumulative figure: 44 of $65 \mathrm{ARD}^{101214}$ ). The incidence of retinal detachment in myopic cataract extractions varies from $7 \%$ to $40 \% .{ }^{910}$ This latter figure comes from a myopic population with a spectacle correction of greater than $-10 \mathrm{D}(42 \%$ of our sample).

The retinal detachment incidence following all extracapsular cataract extractions and posterior vaulted, posterior chamber lenses at the Bromsgrove General Hospital is currently $0 \cdot 167 \%$ (Pearce JL, unpublished data). This is entirely compatible with other large series previously reported $\left(0-2 \cdot 2 \%^{12} 14\right)$ though the very low incidence of this complication in the normal postcataract population makes comparison between published series difficult.

'Clinical' CMO is suspected when an eye, after cataract extraction, suffers a fall in visual acuity one month or more after operation. Such a patient would then undergo fluorescein angiography to confirm the diagnosis. The high incidence of subclinical, thus irrelevant, CMO is not disputed. ${ }^{15-18}$ The 'sealed' primary capsulotomy reduces our 'clinical' CMO incidence to that of intact posterior capsule surgery. With the increasing availability of YAG lasers during 1985 the slightly higher surgical risks of primary capsulotomy have led to a decrease in the indications for its use.

A 2-3 mm stepped scleral lamellar flap with a 10 $\mathrm{mm}$ chord length is used in all extracapsular surgery

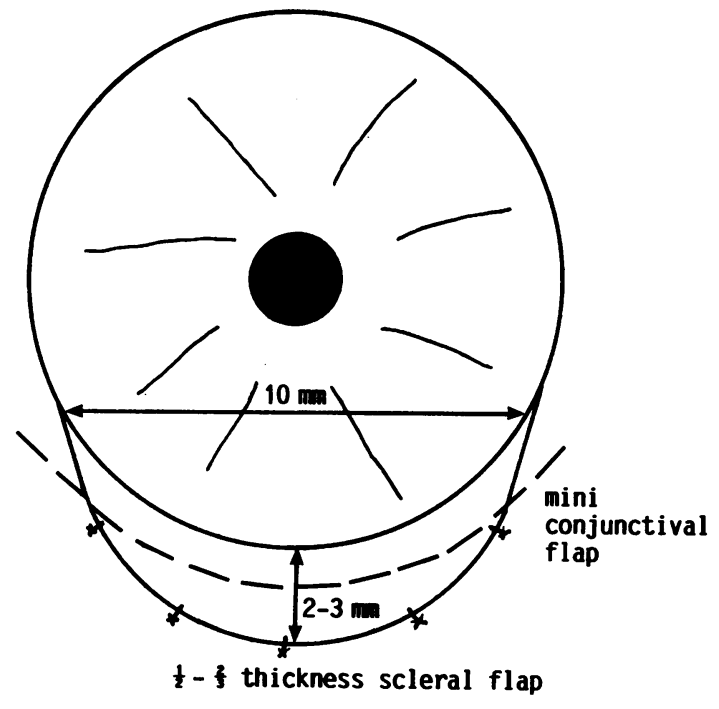

STEPPED SCLERAL CRESCENT INCISION

Fig. 7 Surgical incision and closure used routinely in this population. Notable features are the increased distance from the cornea, very short bite sutures, and obvious potential for combined cataract/drainage surgery.

at this unit (Fig. 7). Extremely short radius, deep 10/0 nylon sutures are employed to achieve a consistently low cylinder while providing a secure wound. The surgical astigmatism achieved with this section compares well even with series designed to show the advantages of using operating keratometers. ${ }^{19}$

\section{CONCLUSION}

The use even of low power, capsule-fixated intraocular lenses may be considered for all myopic cataract extractions. They have few additional complications associated with their use over those of cataract surgery alone. ${ }^{20}$ Their possible advantages include: the pronounced preference of patients for mild myopic refraction over even low grade aphakic hypermetropia ${ }^{21}$; the avoidance of aphakic visual field distortion in patients whose macular function is already compromised; and finally the theoretical protection against aphakic retinal detachment afforded by a near physiological posterior capsule convexity. This latter contention is supported by our series and others. ${ }^{321}$

We acknowledge with thanks the expert advice of Mr M J RoperHall and Mr R Brown, and thank Mrs D Thomas for the preparation of the illustrations.

\section{References}

1 Irvine $\mathbf{A}$. The pathogenesis of aphakic retinal detachment. Ophthalmic Surg 1985; 16: 101-7. 
2 Pearce J. Modern simple extracapsular surgery, with discussion. Trans Ophthalmol Soc UK 1979; 99: 176-82.

3 Percival S. Long term complications from extracapsular cataract surgery. Trans Ophthalmol Soc UK 1985; 104: 915-8.

4 Beetham W, Aiello L, Balodimos M, Koncz L. Ruby laser photocoagulation of early diabetic neovascular retinopathy. Arch Ophthalmol 1970; 83: 261-72.

5 Jain I, Luthra C, Das T. Diabetic retinopathy and its relation to errors of refraction. Arch Ophthalmol 1967; 77: 59-60.

6 Sanders D, Kraff M. Intraocular lens implantation. St Louis: Mosby, 1984: Ch. 21: 216-29.

7 Ho P, Tolentino F. Retinal detachment following extracapsular cataract extraction and posterior chamber intraocular lens implantation. Br J Ophthalmol 1985; 69: 650-3.

8 Fagadau W, Maumenee E, Stark W, Datiles M. Posterior chamber intraocular lenses at the Wilmer Institute: a comparative analysis of complications and visual results. BrJ Ophthalmol 1984; 68: 13-8.

9 Hyams S, Bialik M, Neumann E. Myopia-aphakia: prevalence of retinal detachment. Br J Ophthalmol 1975; 59: 480-2.

10 Ruben M, Rajpurohit P. Distribution of myopia in aphakic retinal detachments. Br J Ophthalmol 1976; 60: 517-21.

11 Clayman H, Jaffe N, Light D, Jaffe M, Cassady J. Intraocular lenses, axial length, and retinal detachment. Am J Ophthalmol 1981; 92: 778-80.

12 Percival S, Anand V, Das S. Prevalence of aphakic retinal detachment. Br J Ophthalmol 1983; 67: 43-5.

13 Osterlin S. On the molecular biology of the vitreous in the aphakic eye. Acta Ophthalmol (Kbh) 1977; 55: 353.
14 Coonan P, Fung W, Webster R, Allen A, Abbott R. The incidence of Retinal Detachment following extracapsular cataract extraction-a ten year study. Ophthalmology (Rochester) 1985; 92: 1096-1101.

15 Drews $R$. The present understanding of cystoid macular oedema. Trans Ophthalmol Soc UK 1985; 104: 744-7.

16 Jaffe N, Clayman H, Jaffe M. Cystoid macular edema after intracapsular and extracapsular cataract extraction with and without an intraocular lens. Ophthalmology (Rochester) 1982; 89: 25-9.

17 Chambless W. Incidence of anterior and posterior segment complications in over 3000 cases of extracapsular cataract extraction: intact and open capsules. Am Intraocular Implant Soc J 1985; 11: 146-8.

18 Kraff M, Sanders D, Jampol L, Lieberman H. Effect of primary capsulotomy with extracapsular surgery on the incidence of pseudophakic cystoid macular edema. Am J Ophthalmol 1984; 98: $166-70$.

19 Jacobi K, Strobel J. Control of postoperative astigmatism. Trans Ophthalmol Soc UK 1985; 104: 715-26.

$20 \mathrm{Jaffe} \mathrm{N}$. Current and future concepts of the design of the intraocular lens. Trans Ophthalmol Soc UK 1985; 104: 70314.

21 Verzella F. High myopia: lower intraocular lens in the posterior chamber for optical purposes. Presented at the Canadian Implant Association Tenth annual meeting, Montreal 22-23 June 1984.

Accepted for publication 9 May 1986. 